\title{
引导反钘：大学生安全感缺失的应对策略研究—以江西服装学 院为例
}

曹昊

江西服装学院心理健康教育与咨询中心

DOI:10.32629/er.v3i5.2750

[摘 要] 安全感是人的基本心理需求,缺乏安全感会对一个人在人际交往,环境适应,情绪表达,人格发展等多方面造成不良影响。安全感的形成 源于婴儿阶段与父母的互动反馈,受到外部环境的影响。通过引导外部环境对个体进行良性反馈,对帮助大学生提升安全感有积极作用。

[关键词] 心理健康教育; 安全感; 应对策略; 引导反馈

Guide feedback: A study on coping strategies of college students' lack of security ——Taking Jiangxi Garment College as an example

Hao Cao

JIANGXI INSTITUTE OF FASHION TECHNOLOGY

[Abstract] sense of security is the basic psychological needs of people, and the lack of sense of security will have a negative impact on interpersonal communication, environmental adaptation, emotional expression, personality development and other aspects. The formation of sense of security comes from the interactive feedback with parents in infancy and is influenced by the external environment. By guiding the external environment to give positive feedback to individuals, it plays a positive role in helping college students to improve sense of security.

[Key words] mental health education; sense of security; coping strategies; guiding feedback

\section{1 提升学生安全感对高校心理健康教育有重要意义}

安全感的健全与否对人的行为模式、人格发展具有重大影响, 马斯洛 (Abraham Harold Maslow) 的需求层次理论把安全需求放在仅次于生理需 求的位置上, 可以说安全感是人心理层面最基本的一种需求。

在高校心理咨询中, 缺乏安全感是引发的大学生心理健康问题的一个 重要原因, 以江西服装学院为例, 通过咨询个案的分类统计, 来访学生普遍 存在安全感缺失的情况。2019年1月-2019年12月, 因人格发展问题进行心 理咨询的学生 26 人, 其中有 17 例显示来访学生存在缺乏安全感的状况, 且 缺乏安全感是引发问题的重要原因, 占比高达 $65 \%$ 。在寝室人际关系, 恋爱 情感等常见问题中, 缺乏安全感的学生占比也分别高达 $57 \%$ 、39\%。

当前生活节奏快, 社会竞争压力大, 当学生的安全感缺乏, 相比过去更 加容易出现各种适应性问题。因此, 帮助安全感缺失的学生提升安全感对 高校心理健康教育有重要意义。

\section{2 影响安全感形成的主要原因}

埃里克森 (Erik H Erikson) 认为一个人安全感的形成主要源于幼儿时 期与父母的情绪连结。通过对学生的访谈, 在成长经历中外部环境的差异 也会对学生的安全感产生正面或负面的影响。如一个童年时期并不缺乏安 全感的学生可能在经历校园欺凌, 朋友背叛后出现缺乏安全感的表现。

一个人的心理倾向和行为模式通常是他根据自身特点应对外部环境 的习惯性策略。根据对咨询个案的研究, 我们发现安全感强的学生往往父 母情绪稳定, 积极乐观, 富有耐心, 这样的学生在成长过程中, 更容易信任 他人, 会主动与身边的人积极沟通并释出善意, 他们乐于探索陌生的环境, 倾向对他人的反馈持正面看法。而安全感缺乏的学生, 因为父母情绪波动 大, 沟通时缺乏耐心, 又或在人际交往中受到了如校园欺凌等强烈的负面 反馈。这类学生往往会表现出对陌生环境的抗拒, 不愿主动与他人沟通, 倾向对他人的反馈持怀疑态度。
对比两类学生的成长经历可知, 安全感的形成主要源自外部环境对个 体释放信号的反馈, 如果个体释放的信号在大部分情况下得到了良好的反 馈, 则该个体的安全感较高, 反之, 就会造成安全感的缺失。在幼儿时期, 人们传递信号的主要对象是父母, 所以来自父母的反馈形成了幼儿对外部 环境的认知, 并形成了相对固定的应对策略。这个阶段形成的应对策略影 响深远, 但并非一成不变, 在之后的成长经历中, 如果外部环境对个体释放 的信号存在明显的倾向性, 同样会对一个人的安全感造成影响。

\begin{tabular}{|c|c|c|}
\hline \multicolumn{3}{|c|}{ 对 44 名缺乏安全雪学生的访谈统计 } \\
\hline 造成安全恶缺失的原因 & 人数 & 占比 \\
\hline 父母关系不睦,经常吵架 & 13 & $29 \%$ \\
\hline 单亲家庭与留守儿童 & 7 & $16 \%$ \\
\hline 父昩管数祖暴或冷漢 & 11 & $25 \%$ \\
\hline 曾受到校园欺凌或朋友背瓶 & 8 & $18 \%$ \\
\hline 外悸, 能力, 经济水平等原因 & 5 & $11 \%$ \\
\hline
\end{tabular}

\section{3 大学生缺乏安全感的常见表现}

不同的个体在缺乏安全感时, 为了能维持心理平衡, 保护自身免受伤 害, 会因气质性格的差异和所处环境的不同形成不同的心理防御机制, 在 这些心理防御机制的作用下, 缺乏安全感的学生容易在适应环境, 人际交 往等方面出现问题, 其中常见的表现有:

3. 1 回避与孤独

回避是在缺乏安全感的学生中最常见的一种表现, 这种表现的内在逻 辑是, 如果我与外界沟通, 总是会受到伤害性的反馈, 那么就尽量减少与外 界的沟通, 自然可以避免受到伤害。在这种策略的影响下, 学生会表现出孤 僻, 不合群的特点, 回避正常的人际交往, 不能很好的参与集体活动。

3. 2 逢迎与爆发

在缺乏安全感的学生中, 有不少是讨好型人格, 他们希望通过抑制自 身需求去曲意逢迎, 从而达到避免触怒他人的目的, 严重者会不惜通过贬 
低自己, 损害自身利益去满足他人。这类学生通常十分敏感, 他们会过度 重视别人的反应, 因为不会拒绝, 缺乏对自我需求的关注, 他们的生活往 往处于长时间的紧张中。值得注意的是, 持有这种策略的学生, 容易受到 负面情绪的影响, 往往负面情绪积累到一定程度时, 会出现伤人或自伤 的极端行为。

3. 3 消极处世

有些学生会通过降低对外界反馈的期待来进行自我保护,他们暗示自 己凡事都是没有价值的, 遇到什么事都不值得生气或悲伤, 表现出对任何 事情都无所谓的态度。这类学生粗看起来比较酒脱, 但深入接触后就会发 现他们对生活缺乏热情, 得过且过, 缺少一般青年应有的活力。

3. 4 怀疑与攻击性

因为在过去的成长经历中, 经常受到负面反馈, 所以缺乏安全感的学 生在面对他人给出的善意反馈时总是难以欣然接受, 他们会下意识的认为 他人都是别有用心的, “他请我吃饭是不是要我帮他做事? ” “老师上课点 我提问是不是在针对我？”诸如此类的想法一旦形成, 就会开始收集证实 这些想法的证据来说服自己, 进而表现出较强的攻击性。这类学生在日常 相处时很难沟通, 防卫心强, 同学, 辅导员通常会感到这类学生性情古怪, 心胸狭隘, 难以理喻, 是思想存在问题, 其实根源在于安全感的缺失。

3. 5 超强的控制欲

如果说前面几种表现是被动的防御, 还有另一种主动防御的情况, 即 试图通过控制外部环境来避免自身可能受到的伤害。这种类型的学生往往 在他人眼里作风十分强势, 凡事勇于出击, 非常自信。实则他们的心理活动 是: “我知道他人想要什么, 但我不能顺从, 如果我不能处于主导地位, 就会 感到焦虑不安, 我必须让他放弃自己的观点, 所有的行为都在我的控制中, 我才能感到安心, 如果对方不顺从我, 我会感到自己不被重视, 必须用更强 硬的方式让对方屈服, 否则我就应该攻击或远离这个人。”经统计, 在恋爱 中因缺乏安全感产生的问题大部分是这种情况。

\section{4 通过引导反馈提升安全感}

如前所述, 缺乏安全感对学生的人格成长, 未来发展都有显而易见的 负面影响。而安全感又源自外部环境对个体的反馈倾向。所以可以通过引 导外部环境的反馈倾向来达到增强安全感的目的。

4. 1 实施方法

想要稳定的获得外部环境的良性反馈, 需要通过对施加反馈的对象进 行引导。具体实施可以分为三步走:

4. 1. 1 学会从对方的视角看问题

为什么付出了这么多却得不到期望中的反馈, 这是缺乏安全感的人经 常碰到的一个问题。通过带给其他人愉悦的感受来收获良性反馈的本身并 没有问题, 问题在于大部分情况下, 我们的付出并没有如想象中那般给他 人带去了愉悦。在与来访学生的访谈中, 经常听到类似 “他们晚上打游戏 到很晚不关灯, 我为了寝室关系和谐一直没提出抗议, 结果他们得寸进
尺。” “刚开学时我为了同学关系和睦主动帮室友带饭, 结果他们现在天天 叫我带饭, 太欺负人了!” “我经常主动帮助他们, 为什么他们还是不喜欢 我?”这样的话。这些同学倾向用自己的感受作为衡量标准。但现实中这 种感受并不被别人认可, 自然也就不会给出期待中的反馈。造成这样的原 因就是因为没有学会站在他人的视角看问题。

4.1.2根据自身特点凸显优势

很多时候, 我们无法了解别人的需求是什么, 那么这种情况下可以根 据自身的特点进行反馈的引导。每个人都是独特的, 拥有属于自己的性格 特征, 而每一种性格特征都能提炼出被大众认可的优点。比如敏感的人通 常细淢, 拘谨的人通常认真。当我们找到适合发挥自身性格优点的事情加 以实施, 并有意识的为自己树立一个能够发挥自身优势的定位, 外部反馈 通常都会呈积极倾向。

4.1.3根据社会文化习俗定制反馈

如果一时无法找出自身性格的优势点, 那么还可以根据所处环境的普 遍共识和文化习俗来定制反馈, 并借由定制的反馈提升自信以达到增强安 全感的目的。例如上课时认真听讲, 会有极大概率获得老师的赞赏, 这就可 以视为一个定制的反馈, 即做出认真听讲的行为必然会得到老师的肯定。

具体操作可以根据自己生活的实际情况, 列出一张表, 上面罗列20-30 件可行的, 做了就有极大概率获得积极反馈的事, 然后给自己定一个目标, 每天在表上选取 $3-5$ 件事来完成。通过这样不断的接收定制后的反馈, 不断 巩固自己是受欢迎的, 是被喜爱的这种观念, 当获得良性反馈成为常态, 就 能达到提升安全感的效果。

4. 2 效果与结论

选取了 15 名被试采用引导反馈法, 其中 10 名有寝室矛盾问题、 5 名恋爱 情感问题。通过 3 个月的跟踪观察, 10 名存在寝室矛盾的被试中有 5 人表示 寝室关系有明显改善, 3 人表示有一定改善, 5 名存在恋爱情感问题的被试 中有 2 人表示有较明显的效果。

根据测试结果可知引导反馈法对改善因安全感缺失出现的人格发展 问题, 尤其是在改善人际关系方面有较好的效果。对提升大学生心理健康 具有积极意义。

[参考文献]

$[1][$ 美]亚伯拉罕・马斯洛,许金声译.动机与人格(第三版) [M].中国人 民大学出版社,2007.

[2][美]埃里克.H.埃里克森,孙名之译.同一性:青少年与危机[M].中央 编译出版社,2015.

[3]高峰强,赵锦哲,杨华勇,等.差怯对攻击的影响:安全感和社会比较 倾向的中介作用[J].心理学探新,2019,39(04):358-362.

作者简介:

曹昊(1986--), 男, 汉族, 江西南昌人, 大学本科, 助教, 研究方向: 大 学生心理健康教育。 